\title{
Aquatic Antagonists: Sea Cucumbers (Holothuroidea)
}

\author{
Carter R. Ellis, MD; Dirk M. Elston, MD; Juan Pedro Lonza Joustra, MD; Vidal Haddad Jr, MD
}

\section{PRACTICE POINTS}

- Sea cucumbers produce a toxin known as holothurin, which is contained in specialized structures called Cuvierian tubules and secreted onto the outer surface of the body wall. Some species also eject portions of their toxic inner organs through the anus as a defensive mechanism.

- In humans, the holothurin toxins cause an acute irritant dermatitis upon contact with the skin and a pain ful chemical conjunctivitis upon contact with the eyes.

- In addition to their own toxin, sea cucumbers also can secrete undigested nematocysts of previously consumed cnidarians through their integument, causing additional effects on human skin.

- The dermatologic effects of sea cucumbers can be prevented with the use of gloves and swim masks or goggles.
Sea cucumbers are sausage-shaped aquatic creatures living on the ocean floors across the world. They are considered a food delicacy in many countries but also are known to have many beneficial pharmaceutical properties; however, sea cucumbers also contain a dangerous component called holothurin, which has been shown to have a negative impact on the health of both humans and other wildlife. In humans, exposure to holothurin can cause a painful irritant dermatitis, irritation of the mucous membranes, potential blindness, and, if ingested, even possible death. Treatment options include irrigation with seawater, soap, isopropyl alcohol, and vinegar.

Cutis. 2021;108:68-70. $\bigcirc$ ea cucumbers - commonly known as trepang in Indonesia, namako in Japan, and hai shen in China, where they are treasured as a food delicacy-are sea creatures belonging to the phylum Echinodermata, class Holothuridea, and family Cucumariidae. ${ }^{1,2}$ They are an integral part of a variety of marine habitats, serving as cleaners as they filter through sediment for nutrients. They can be found on the ocean floor under hundreds of feet of water or in shallow sandy waters along the coast, but they most commonly are found living among coral reefs. Sea cucumbers look just as they sound-shaped like cucumbers or sausages, ranging from under 1 inch to upwards of 6 feet in length depending on the specific species (Figure 1). They have a group of tentacles around the mouth used for filtering sediment, and they move about the ocean floor on tubular feet protruding through the body wall, similar to a sea star.

\section{Beneficial Properties and Cultural Relevance}

Although more than 1200 species of sea cucumbers have been identified thus far, only about 20 of these are edible. ${ }^{2}$ The most common of the edible species is Stichopus japonicus, which can be found off the coasts of Korea, China, Japan, and Russia. This particular species most commonly is used in traditional dishes and is divided into 3 groups based on the color: red, green, or black. The price and taste of sea cucumbers varies based on the color, with red being the most expensive. ${ }^{2}$ The body wall of the sea cucumber is cleaned, repeatedly boiled, and dried until edible. It is considered a delicacy, not only in food but also in pharmaceutical forms, as it is comprised

Drs. Ellis and Elston are from the Medical University of South Carolina, Charleston. Dr. Lonza Joustra is in independent practice, Iquique, Chile. Dr. Haddad is from the Department of Dermatology, Botucatu School of Medicine, Brazil.

The authors report no conflict of interest.

Correspondence: Dirk M. Elston, MD (elston@musc.edu).

doi: 10.12788 /cutis.0323 

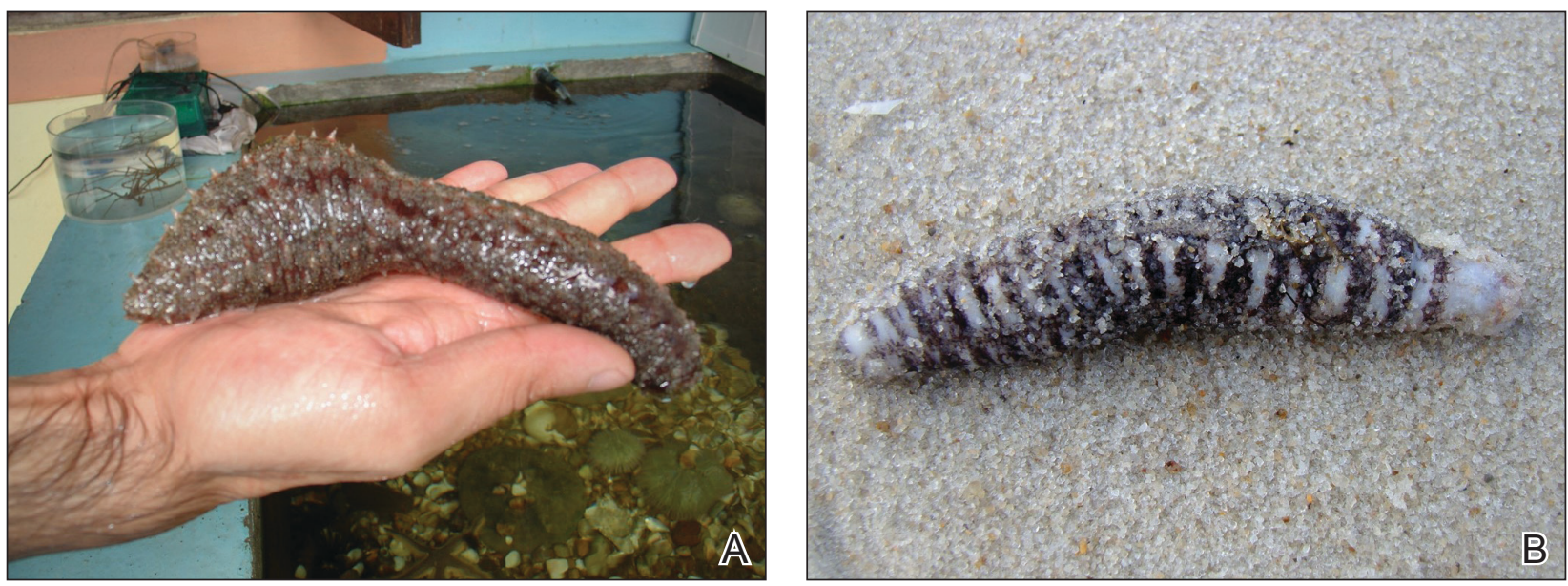

FIGURE 1. A and B, Sea cucumbers (Cucumariidae family). Photographs courtesy of Vidal Haddad Jr, MD.

of a variety of vitamins, minerals, and other nutrients that are thought to provide anticancer, anticoagulant, antioxidant, antifungal, and anti-inflammatory properties. Components of the body wall include collagen, mucopolysaccharides, peptides, gelatin, glycosaminoglycans, glycosides (including various holotoxins), hydroxylates, saponins, and fatty acids. ${ }^{2}$ The regenerative properties of the sea cucumber also are important in future biomedical developments.

\section{Toxic Properties}

Although sea cucumbers have proven to have many beneficial properties, at least 30 species also produce potent toxins that pose a danger to both humans and other wildlife. ${ }^{3}$ The toxins are collectively referred to as holothurin; however, specific species actually produce a variety of holothurin toxins with unique chemical structures. Each toxin is a variation of a specific triterpene glycoside called saponins, which are common glycosides in the plant world. Holothurin was the first saponin to be found in animals. The only animals known to contain holothurin are the echinoderms, including sea cucumbers and sea stars. ${ }^{1}$ Holothurins A and B are the 2 groups of holothurin toxins produced specifically by sea cucumbers. The toxins are composed of roughly $60 \%$ glycosides and pigment; $30 \%$ free amino acids (alanine, arginine, cysteine, glycine, glutamic acid, histidine, serine, and valine); $5 \%$ to $10 \%$ insoluble proteins; and $1 \%$ cholesterol, salts, and polypeptides. ${ }^{3}$

Holothurins are concentrated in granules within specialized structures of the sea cucumber called Cuvierian tubules, which freely float in the posterior coelomic cavity of the sea cucumber and are attached at the base of the respiratory tree. It is with these tubules that sea cucumbers utilize a unique defensive mechanism. Upon disturbance, the sea cucumber will turn its posterior end to the threat and squeeze its body in a series of violent contractions, inducing a tear in the cloacal wall. ${ }^{4}$
The tubules pass through this tear, are autotomized from the attachment point at the respiratory tree, and are finally expelled through the anus onto the predator and into the surrounding waters. The tubules are both sticky on contact and poisonous due to the holothurin, allowing the sea cucumber to crawl away from the threat unscathed. Over time, the tubules will regenerate, allowing the sea cucumber to protect itself again in the face of future danger.

Aside from direct disturbance by a threat, sea cucumbers also are known to undergo evisceration due to high temperatures and oxygen deficiency. ${ }^{3}$ Species that lack Cuvierian tubules can still produce holothurin toxins, though the toxins are secreted onto the outer surface of the body wall and mainly pose a risk with direct contact undiluted by seawater. ${ }^{5}$ The toxin induces a neural blockade in other sea creatures through its interaction with ion channels. On Asian islands, sea cucumbers have been exploited for this ability and commonly are thrown into tidal pools by fishermen to paralyze fish for easier capture. ${ }^{1}$

\section{Effects on Human Skin}

In humans, the holothurin toxins of sea cucumbers cause an acute irritant dermatitis upon contact with the skin. ${ }^{6}$ Fishermen or divers handling sea cucumbers without gloves may present with an irritant contact dermatitis characterized by marked erythema and swelling (Figure 2).$^{6-8}$ Additionally, holothurin toxins can cause irritation of the mucous membranes of the eyes and mouth. Contact with the mucous membranes of the eyes can induce a painful conjunctivitis that may result in blindness. ${ }^{6,8}$ Ingestion of large quantities of sea cucumber can produce an anticoagulant effect, and toxins in some species act similar to cardiac glycosides., ${ }^{3,9}$

In addition to their own toxins, sea cucumbers also can secrete undigested nematocysts of previously consumed cnidarians through the integument. ${ }^{7,10}$ In this case, 

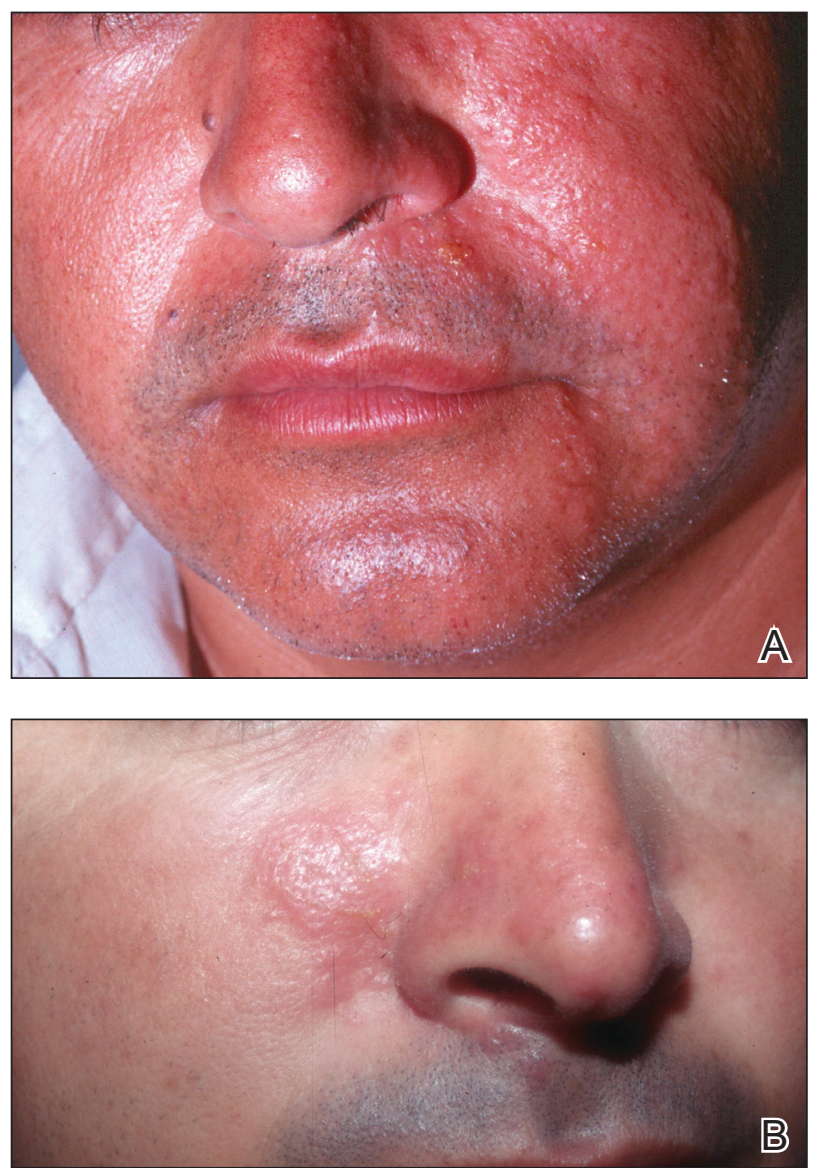

FIGURE 2. A and B, Irritant dermatitis of the face caused by holothurin toxin released by a sea cucumber. Photographs courtesy of Juan Pedro Lonza Joustra, MD.

the result of direct contact with the body wall is similar to a jellyfish sting in addition to the irritant contact dermatitis caused by the holothurin toxin.

\section{Treatment and Prevention}

Irritant dermatitis resulting from contact with a holothurin toxin is first treated with cleansing of the affected area at the time of exposure with generous amounts of seawater or preferably hot seawater and soap. Most marine toxins are inactivated by heat, but holothurin is partially heat stable. Vinegar or isopropyl alcohol also have been used. ${ }^{9}$ The result is removal of the slime containing the holothurin toxin rather than deactivation of the toxin. Although this alone may relieve symptoms, dermatitis also may be addressed with topical anesthetics, corticosteroids, or, if a severe reaction has occurred, systemic steroids. ${ }^{9}$
Conjunctivitis should be addressed with copious irrigation with tap water and topical anesthesia. Following proper irrigation, providers may choose to follow up with fluorescein staining to rule out corneal injury. ${ }^{10}$

The dermatologic effects of holothurin toxins can be prevented with the use of gloves and diving masks or goggles. Proper protective wear should be utilized not only when directly handling sea cucumbers but also when swimming in water where sea cucumbers may be present. Systemic toxicity can be prevented by proper cooking, as holothurin toxins are only partially heat resistant and also are hydrolyzed into nontoxic products by gastric acid. Additionally, the species of the sea cucumber should be confirmed prior to consumption, as edible species are known to contain less toxin.

\section{Conclusion}

Although sea cucumbers have ecologic, culinary, and pharmaceutical value, they also can pose a threat to both humans and wildlife. The holothurin toxins produced by sea cucumbers cause a painful contact dermatitis and can lead to conjunctivitis and even blindness following eye exposure. Although the toxin is broken down into nontoxic metabolites by gastric acid, large amounts of potent variants can induce systemic effects. Individuals who come in contact with sea cucumbers, such as fishermen and divers, should utilize proper protection including gloves and protective eyewear.

\section{REFERENCES}

1. Burnett K, Fenner P, Williamson J. Venomous and Poisonous Marine Animals: A Medical and Biological Handbook. University of New South Wales Press; 1996.

2. Oh GW, Ko SC, Lee DH, et al. Biological activities and biomedical potential of sea cucumber (Stichopus japonicus): a review. Fisheries Aquatic Sci. 2017;20:28.

3. Nigrelli RF, Jakowska S. Effects of holothurian, a steroid saponin from the Bahamian sea cucumber (Actinopyga agassizi), on various biological systems. Ann NY Acad Sci. 1960;90:884-892.

4. Demeuldre M, Hennebert E, Bonneel M, et al. Mechanical adaptability of sea cucumber Cuvierian tubules involves a mutable collagenous tissue. J Exp Biol. 2017;220:2108-2119.

5. Matranga V, ed. Echinodermata: Progress in Molecular and Subcellular Biology. Springer; 2005.

6. Tlougan, BE, Podjasek, JO, Adams BB. Aquatic sports dermatoses. part 2-in the water: saltwater dermatoses. Int J Dermatol. 2010; 49:994-1002

7. Bonamonte D, Verni P, Filoni A, et al. Dermatitis caused by echinoderms. In: Bonamonte D, Angelini G, eds. Springer; 2016:59-72.

8. Haddad V Jr. Medical Emergencies Caused by Aquatic Animals: A Zoological and Clinical Guide. Springer International Publishing; 2016.

9. French LK, Horowitz BZ. Marine vertebrates, cnidarians, and mollusks. In: Brent J, Burkhart K, Dargan P, et al, eds. Critical Care Toxicology. Springer; 2017:1-30.

10. Smith ML. Skin problems from marine echinoderms. Dermatol Ther. 2002;15:30-33. 